\title{
Membrane fatty acids as phenotypic markers in the polyphasic taxonomy of methylotrophs within the Proteobacteria
}

\author{
James B. GuCKert, ${ }^{1 *} \dagger$ David B. Ringelberg, ${ }^{1}$ David C. White,,${ }^{1,2}$ Richard S. HANSON ${ }^{3}$ and \\ BONNIE J. BRATINA \\ ${ }^{1}$ Institute for Applied Microbiology, University of Tennessee, 10515 Research Dr. Suite 300, Knoxville, \\ Tennessee 37932-2567, USA \\ ${ }^{2}$ Department of Microbiology, University of Tennessee, Knoxville, Tennessee 37932-2567, USA and \\ Environmental Sciences Division, Oak Ridge National Laboratory, Oak Ridge, Tennessee 37831, USA \\ ${ }^{3}$ Gray Freshwater Biological Institute, University of Minnesota, Navarre, Minnesota 55392, USA
}

(Received 27 June 1991; accepted 25 July 1991)

\begin{abstract}
A polyphasic approach to bacterial taxonomy attempts to integrate phylogenetic relationships with phenotypic marker analysis. This study describes the application of membrane fatty acids as a phenotypic marker for methylotrophs. Detailed phospholipid, ester-linked fatty acid (PLFA) profiles are reported for 17 methylotrophic eubacterial strains. These profiles included verification of double bond positions and geometries, both critical features for this analysis. Multivariate cluster analysis was used to indicate groupings of these strains along with literature values of both methylotrophs and non-methylotrophs based on the PLFA phenotype. Like many phenotypic characteristics, PLFA profiles were influenced by environmental conditions. The instabilities displayed, however, were predictable from physiological studies including increased trans/cis and cyclopropyl/cis ratios. Cluster analysis of PLFA profiles generated by separate investigators with different culture conditions indicated reproducibility by strain and species. The PLFA phenotype relationships compare favourably with phylogenetic associations based on 16S rRNA data for methylotrophs and will continue to be a valuable phenotypic marker for Proteobacteria taxonomy.
\end{abstract}

\section{Introduction}

Studies of bacterial evolution are utilizing nucleic acid sequence analyses to propose new phylogenetic relationships which were not previously recognized with traditional classification techniques (Woese, 1987). This is especially true within the diverse Gram-negative eubacterial species once referred to as the purple bacteria and now termed the Proteobacteria (Murray et al., 1990). Woese (1987) reminds microbiologists and evolutionary biologists, however, that phylogenies derived from such sequence data must not be over-interpreted. He suggests that these relationships be viewed as hypotheses to be tested and either strengthened or rejected using independent methodology. A recent report has re-emphasized

† Present address: Environmental Safety Department, The Procter and Gamble Company, Ivorydale Technical Center, 5299 Spring Grove Ave, Cincinnati, Ohio 45217-1087, USA.

Abbreviations: ECL, equivalent chain length; FAME, fatty acid methyl esters; PLFA, phospholipid, ester-linked fatty acid; SV, similarity value. the importance of a polyphasic taxonomic approach to Proteobacteria taxonomy, that is, the integrated use of both phylogenetic and phenotypic characteristics to test for evolutionary relationships (Murray et al., 1990).

There are many diverse approaches to microbial chemotaxonomy. Chemical profiling techniques using chromatography or electrophoresis have been a common approach, and many such techniques have been recently reviewed (Goodfellow \& Minnikin, 1985; Brondz \& Olsen, 1986). Bacterial fatty acid analyses are one of the more popular profiling methods in chemotaxonomy (Tornabene, 1985).

For over a decade, this laboratory has been involved in microbial community structure analysis using membrane fatty acid profiles as a reproducible and quantitative method of investigating in situ ecological relationships (e.g. White et al., 1979a, b; Guckert et al., 1985; Guckert \& White, 1988; Ringelberg et al., 1989). Owing to the complexity of these fatty acid profiles, including multiple positional and geometric isomers of unsaturated fatty acids (e.g. Guckert et al., 1986; Ringelberg et al., 1989), 
we have also been very sensitive to the analytical quality of these profiles, adapting many analytical verification procedures to these studies (e.g. Nichols et al., 1986a; Guckert et al., 1987).

Previous research in our laboratory indicated that one group within the Proteobacteria, the methylotrophs, had very diverse membrane fatty acid profiles with several unusual monounsaturated isomers (Nichols et al., 1985; Jahnke \& Nichols, 1986; Ringelberg et al., 1989). The systematics of the methylotrophs is currently based on nucleic acid sequencing assays (Tsuji et al., 1990; Bulygina et al., 1990). This present study reports membrane fatty acid profiles for 17 methylotrophic eubacterial strains of which many have been placed on 16S rRNA phylogenetic trees (Tsuji et al., 1990). In addition, we report groupings of these strains based on fatty acid profiles as a phenotypic marker, and these groupings are discussed in relation to literature profiles for both methylotrophs and non-methylotrophs along with their reported phylogenetic relationships. Discussions of membrane fatty acids as reproducible and stable phenotypic markers, as well as the use of these in a polyphasic approach to Proteobacteria taxonomy, especially for the methylotrophs, are also given.

\section{Methods}

Micro-organisms. Methylotrophic cultures were obtained as freezedried pellets from B. J. Bratina and R. S. Hanson of the Gray Freshwater Biological Institute, University of Minnesota. Descriptions of these strains are given in Table 1. Details of the origins of these strains are available in Tsuji et al. (1990), Tsien et al. (1990) or from R. S. Hanson. 16S rRNA phylogenetic trees have been produced which include nine of these strains (Tsuji et al., 1990). The remaining strains are currently being sequenced for inclusion (Tsien et al., 1990; B. J. Bratina \& R. S. Hanson, unpublished). Agrobacterium tumefaciens (ATCC 33970) was obtained as a Preceptrol culture.

Culture conditions. Methylotrophic bacteria were grown as described by Tsuji et al. (1990) and Tsien et al. (1990) in a minimal salts medium (Dalton \& Whittenbury, 1976) with either methane or methanol as the sole carbon source. In most cases, only one culture was analysed for each strain. Two independent replicates of the strain Methylocystis parvus OBBP were prepared. These replicates are abbreviated OBBP1 and OBBP2 in this study. Replicate cultures of Methylosinus trichosporium OB3b were also grown in a $1 \mu \mathrm{M}$-copper-supplemented medium to test for copper-induced membrane fatty acid changes. These replicates are abbreviated OB3bla and OB3blb for the $1 \mu \mathrm{M}-$, and $\mathrm{OB} 3 \mathrm{~b} 0 \mathrm{a}$ and $\mathrm{OB} 3 \mathrm{~b} 0 \mathrm{~b}$ for the $0 \mu \mathrm{M}$-copper treatments, respectively. A. tumefaciens was grown at the University of Tennessee in Trypticase Soy Broth (BBL 11768, ATCC medium 18). Incubations to obtain cell mass for lipid extractions were conducted with continuous shaking at $27^{\circ} \mathrm{C}$ for both $16 \mathrm{~h}$ (three replicates, exponential growth phase) and 64-120 hours (four replicates, stationary growth phase). This species is abbreviated AGRO in this study.

Extraction and preparation of membrane lipids. The equivalent of $20-25 \mathrm{mg}$ dry weight of bacterial cells as extracted in a Bligh \& Dyer (1959) single-phase solvent system modified to include phosphate buffer (White et al., 1979b). During all procedures, care was exercised
Table 1. Descriptions of methylotrophic strains analysed in this study

The pathway indicated is used to assimilate formaldehyde. Ribulose monophosphate pathway methylotrophs are historically referred to as 'type I' methylotrophs and serine pathway methylotrophs as 'type II' methylotrophs. Abbreviations given are used throughout this paper. Details of strains are available in Tsuji et al. (1990), Tsien et al. (1990) and references contained therein.

\begin{tabular}{lll}
\hline \hline \multicolumn{1}{c}{ Strain } & Pigments & Abbreviation \\
\hline $\begin{array}{l}\text { Ribulose monophosphate pathway } \\
\text { methanotrophs }\end{array}$ & \\
Methylococcus capsulatus BATH & & \\
Methylococcus luteus & Yellow & MCAP \\
Methylomonas methanica & Pink & MMET \\
Methylomonas gracilis & Brown & MGRA \\
Ribulose monophosphate pathway & & \\
methylotrophs unable to use methane & & \\
Methylobacillus glycogenes & & MGLY \\
Methylomonas methanolica & & MLIC \\
Methylomonas methylovora & & MLOV \\
Methylophilus methylotrophus AS1 & & AS1 \\
Methylotrophic sp. DM11 & DM11 \\
Serine pathway methanotrophs & & \\
Methylosinus trichosporium OB3b & & OB3b \\
Methylosinus methanica $81 Z$ & $81 Z$ \\
Methylosinus sp. B & & SIN B \\
Methylocystis parvus OBBP & & OBBP \\
Serine pathway methylotrophs unable & & \\
to use methane & & \\
Methylobacterium organophilum XX & Pink & XX \\
Methylobacterium extorquens AM1 & Pink & AM1 \\
Methylobacterium sp. DM4 & Pink & DM4 \\
Hyphomicrobium sp. DM2 & & DM2 \\
\hline \hline
\end{tabular}

to minimize both contamination and artifactual changes in lipid structures using procedures recommended in Guckert \& White (1988). Cells were extracted at room temperature in $142.5 \mathrm{ml}$ chloroform/ methanol/potassium phosphate buffer $(1: 2: 0 \cdot 8$ by vol.; $50 \mathrm{mM}, \mathrm{pH} 7 \cdot 4)$ for $3 \mathrm{~h}$, at which time $37.5 \mathrm{ml}$ each of chloroform and distilled water were added to separate the aqueous (upper) and organic (lower) phases overnight. The organic phase (containing the bacterial lipids) was collected and the solvent removed with a rotary evaporator at $37^{\circ} \mathrm{C}$. The total lipid extract was separated into lipid classes by silicic acid column chromatography as detailed in Guckert et al. (1985). The phospholipid-containing methanol fraction was further used in the evaluation of bacterial membrane lipid profiles. The phospholipid, ester-linked fatty acids (PLFAs) were prepared for gas chromatography (GC) analysis by a mild alkaline transesterification (Guckert $\boldsymbol{e t}$ al., 1985). The resultant fatty acid methyl esters (FAME) were separated, quantified and tentatively identified by capillary GC using a $50 \mathrm{~m} \mathrm{HP}-1$ (non-polar methyl silicone, Hewlett-Packard) column using the conditions described by Ringelberg et al. (1989). FAME structure was verified by $\mathrm{GC} /$ mass spectrometry (MS) as described in Ringelberg et al. (1989). Bacterial fatty acid double bond position and geometry was confirmed using GC/MS analysis of the dimethyl disulphide adducts of the monounsaturated FAME as described by Nichols et al. (1986a). Cyclopropyl FAME ring positions were determined by GC/MS analysis of hydrogenation products as reviewed by Guckert et al. (1985). Additional verification was done, as required, by equivalent chain length (ECL) analysis (Christie, 1989) with separations on several capillary GC column stationary phases 
incluidng HP-1 (Hewlett-Packard), Rtx-1 (non-polar dimethyl polysiloxane, Restek) and Rtx-225 (polar 50\% cyanopropylmethyl $/ 50 \%$ phenylmethyl polysiloxane, Restek).

Fatty acid nomenclature. Fatty acids are designated as $\mathrm{A}: \mathrm{B} \omega \mathrm{C}$, where $\mathrm{A}$ is the total number of carbon atoms, $\mathrm{B}$ is the number of double bonds, and $C$ is the position of the double bond from the aliphatic $(\omega)$ end of the molecule. Geometry of this bond is indicated as ' $\mathrm{c}$ ' for cis and ' $t$ ' for trans. The prefixes ' $\mathrm{i}$ ' and ' $\mathrm{a}$ ' refer to iso and anteiso methylbranching, respectively (Kates, 1986). Cyclopropyl fatty acids are designated as 'cy', with the ring position in parenthesis relative to the aliphatic $(\omega)$ end.

Literature sources of methylotrophic fatty acid profiles. Any successful phenotypic marker for chemotaxonomic studies must be directly comparable with literature values. Part of the data analysis presented in this study is a comparison of the PLFA profiles from the methylotrophs listed in Table 1 with similar and identical strain PLFA profiles available in the literature. Not all published methylotrophic PLFA profiles, however, are analytically precise enough to be included into a rigorous multivariate statistical analysis (see Wold et al., 1984). For instance, the membrane fatty acid profile for Methylosinus trichosporium OB3b was first published in 1975 (Weaver et al., 1975); however, no $16: 1$ or $18: 1$ isomers were separated. Nichols et al. (1985) reported that this same strain had several $18: 1$ isomers with $18: 1 \omega 8 \mathrm{c}$, $18: 1 \omega 7 \mathrm{c}$, and $18: 1 \omega 8 \mathrm{t}$ accounting for over $80 \%$ of the entire PLFA profile. The non-resolved data set of Weaver et al. (1975) is clearly not appropriate for broad chemotaxonomic studies.

Literature fatty acid profiles of sufficient quality were found for the following strains: Methylococcus capsulatus BATH, with several replicates grown under both high and low oxygen culture conditions (abbreviated MCAPhi, MCAPlo) (Jahnke \& Nichols, 1986); Methylomonas sp. 761 (abbreviated 761); Methylosinus trichosporium OB3b (abbreviated OB3b); Methylobacterium organophilum XX (grown with either methane or methanol as sole carbon source and abbreviated as XXCH4 or XXMETH, respectively); and Methylobacterium organophilum RG (abbreviated RG) (Nichols et al., 1985).

In addition to the inclusion of methylotrophic lipid profiles from the literature, several non-methylotrophic eubacteria were also included in this analysis. These had been previously assigned to specific divisions within the Proteobacteria using 16S rRNA analysis [after Woese (1987) and Devereux et al. (1989)] and had good-quality PLFA profiles published. These strains included: Vitreoscilla stercoraria and two other Vitreoscilla strains (Nichols et al., 1986b); Legionella sp. (Pfiffner et al., 1988); Desulfovibrio spp. (Edlund et al., 1985); and Desulfobacter spp. (Dowling et al., 1986).

Exponential growth phase Escherichia coli data from a recent review of microbial lipids (Ratledge \& Wilkinson, 1988) were also included. This review did not specify double bond positions; however, this laboratory has verified the double bond and cyclopropyl ring positions for $E$. coli (unpublished data). The abbreviations used for these strains are given in the legend of Fig. 2.

Statistical analysis. All averaged data which include an indication of variability about the mean are shown \pm sample SD. Data used in the calculations of these standard deviations are detailed in Results and Discussion. The bacterial membrane lipid profiles, or more specifically, their PLFA profiles were treated as multivariate data and analysed using cluster analysis. Dendrograms were constructed using a complete linkage, farthest neighbour method with the PC-based software package Ein $*$ Sight (Infometrix). The dendrograms presented are essentially identical to others produced with other clustering algorithms available both through Ein $*$ Sight and the mainframe software package sPSsX (Version 3.0). In addition, the dendrograms were stable to the exclusion of minor $(<0 \cdot 1 \mathrm{~mol} \%)$, unidentified PLFA and highly correlated PLFA. We feel, therefore, that the relationships presented are robust to the method of clustering utilized. The similarity values $(1 \cdot 00=$ identical $)$ given were determined by the Ein $*$ Sight program using modified Euclidean distances and were stable to the inclusion and exclusion of different strains of eubacteria. A conservative minimum similarity value (SV) of 0.65 was used to define a clustered 'group'. Average PLFA profiles were calculated for members of groups or separate clusters within groups.

\section{Results and Discussion}

PLFA profiles are given for the 17 methylotrophic strains in Table 2. There are four unidentified (UNK 1, $2,3,4)$ fatty acids listed in Table 2 . Characteristics of these fatty acids, including ECL values on two GC columns and GC/MS ion information, are given in Table 3. The GC/MS spectra and ECL values on the non-polar Rtx-1 column suggest that these are diunsaturated 18-carbon FAME. The ECL values on the more polar Rtx-225 are not consistent with this identification, with co-elution of these unknowns occurring with monounsaturated 18-carbon FAME. These unknown fatty acids were not shown to be an important discriminating parameter in the multivariate cluster analysis used to determine taxonomy relationships.

PLFA values are given in Table 2 as the percentage of total moles of PLFA for each strain ( $\mathrm{mol} \%)$. The total molar amount of PLFA recovered per gram dry weight of culture material is also given. The average PLFA content for all strains was $114 \pm 87 \mu \mathrm{mol}(\mathrm{g} \mathrm{dry} \mathrm{wt})^{-1}$, which compares favourably to the reported value of $100 \mu \mathrm{mol}$ (g dry wt) ${ }^{-1}$ for 'typical' eubacteria (White et al., 1979a). The range of this value is large [12.08-344.45 $\mu \mathrm{mol}$ (g dry wt) ${ }^{-1}$ ], but this is due to media salts included in cultures during freeze-drying and will not affect the analysis.

\section{Reproducibility of PLFA phenotypes}

All strains in Table 2 are represented by one analysis except for Methylocystis parvus OBBP and Methylosinus trichosporium OB3b for which mean values for the replicates discussed above are given. The PLFA profiles for the methylotrophic strains in Table 2 (excluding the OBBP and OB3b mean values), the two replicates of OBBP (OBBP1 and OBBP2), the four samples of OB3b under different conditions of copper supplementation (OB3b0a, OB3b0b, OB3bla, OB3blb) and the literature methylotroph PLFA profiles were analysed by cluster analysis (Fig. 1; literature values are marked with the symbol ' $\boldsymbol{\square}$ ' on the dendrogram). No effort was made to exactly mimic culture conditions for strains which have been previously analysed and published. In spite of this, replicate strains from different sources were closely clustered, as described below. The dendrogram (Fig. 1) has five groups (SV >0.65), each marked with a star. 
Table 2. PLFA profiles of 17 methylotrophic eubacterial strains

Results are expressed as a percentage of total PLFA recovered. Fatty acids are listed in their order of elution off a non-polar methyl silicone stationary phase (e.g. HP-1 or Rtx-1). Strain abbreviations are defined in Table 1.

\begin{tabular}{|c|c|c|c|c|c|c|c|c|c|c|c|c|c|c|c|c|c|}
\hline Fatty acid & $\mathbf{X X}$ & AMI & DM4 & $81 \mathrm{Z}$ & DM2 & SIN B & OBBP & OB3b & MCAP & MLUT & MGRA & MGLY & DM11 & MLOV & MLIC & AS1 & MMET \\
\hline 4:0 & 0.00 & 0.00 & $0 \cdot 00$ & 0.00 & 0.00 & $0 \cdot 00$ & 0.00 & 0.01 & $2 \cdot 35$ & 3.45 & 0.00 & 0.33 & $1 \cdot 12$ & 0.54 & 0.79 & 1.28 & $20 \cdot 76$ \\
\hline $5: 0$ & 0.00 & 0.00 & 0.00 & 0.00 & 0.00 & 0.00 & 0.00 & 0.00 & $1 \cdot 22$ & 0.00 & $2 \cdot 31$ & 0.20 & 0.00 & 0.30 & 0.17 & 0.00 & $0 \cdot 30$ \\
\hline $16: 1 \omega 8 \mathrm{c}$ & 0.00 & 0.00 & 0.00 & 0.00 & 0.00 & 0.00 & 0.00 & 0.00 & 0.00 & 0.00 & 0.00 & 0.00 & 0.00 & 0.00 & 0.00 & 0.00 & $40 \cdot 90$ \\
\hline $16: 1 \omega 7 c$ & 0.00 & $5 \cdot 34$ & $6 \cdot 41$ & $9 \cdot 90$ & 0.00 & $15 \cdot 19$ & 0.67 & $10 \cdot 71$ & 18.66 & 47.93 & $45 \cdot 20$ & 55.68 & 43.84 & $37 \cdot 21$ & 37.60 & $35 \cdot 47$ & 10.86 \\
\hline $16: 1 \omega 6 c$ & 0.00 & 0.00 & 0.00 & 0.00 & 0.00 & 0.00 & 0.00 & 0.00 & $10 \cdot 26$ & $6 \cdot 26$ & 0.00 & 0.00 & $0 \cdot 00$ & 0.00 & 0.00 & 0.00 & $3 \cdot 35$ \\
\hline $16: 1 \omega 7 t$ & 0.00 & 0.00 & $0 \cdot 00$ & 0.00 & 0.00 & 0.64 & 0.00 & 0.37 & $8 \cdot 54$ & 0.00 & 0.00 & 0.00 & 0.00 & $2 \cdot 08$ & 0.00 & 0.72 & 1.92 \\
\hline $16: 1 \omega 6 t$ & 0.00 & 0.00 & $0 \cdot 00$ & 0.00 & $0 \cdot 00$ & 0.00 & 0.00 & 0.00 & 0.00 & 0.00 & 0.00 & 0.00 & 0.00 & 0.00 & 0.00 & 0.00 & 0.57 \\
\hline $16: 1 \omega 5 c$ & $0 \cdot 00$ & 0.00 & 0.00 & $0 \cdot 00$ & $0 \cdot 00$ & 0.00 & 0.00 & 0.00 & $12 \cdot 36$ & 1.39 & 0.00 & 0.00 & $0 \cdot 00$ & 0.00 & 0.00 & 0.00 & 0.83 \\
\hline $16: 1 \omega 5 t$ & 0.00 & 0.00 & 0.00 & 0.00 & 0.00 & 0.00 & 0.00 & 0.00 & 0.38 & $17 \cdot 79$ & 0.00 & 0.00 & 0.00 & 0.00 & 0.00 & 0.00 & $15 \cdot 57$ \\
\hline $16: 0$ & $6 \cdot 36$ & 4.94 & 3.05 & 1.52 & 3.04 & 1.02 & $0 \cdot 26$ & $1 \cdot 17$ & 32.53 & $15 \cdot 19$ & 52.49 & $36 \cdot 15$ & 39.83 & 42.60 & $41 \cdot 32$ & $43 \cdot 32$ & $4 \cdot 29$ \\
\hline & 0.00 & $0 \cdot 00$ & 0.00 & 0.00 & 0.00 & 0.00 & 0.00 & 0.06 & 0.00 & 0.00 & 0.00 & $0 \cdot 00$ & 0.00 & 0.00 & 0.00 & $0 \cdot 00$ & $0 \cdot 00$ \\
\hline a 17:0 & 0.00 & $0 \cdot 00$ & 0.00 & 0.00 & 0.00 & 0.00 & $0 \cdot 00$ & $0 \cdot 10$ & 0.00 & 0.00 & 0.00 & 0.00 & 0.00 & 0.00 & 0.00 & 0.00 & $0 \cdot 00$ \\
\hline $\operatorname{cy} 17: 0(\omega 7,8)$ & $0 \cdot 00$ & $0 \cdot 00$ & $0 \cdot 00$ & $0 \cdot 26$ & 0.00 & 0.00 & $0 \cdot 00$ & 0.00 & $7 \cdot 37$ & 0.00 & 0.00 & 1.96 & $14 \cdot 52$ & $11 \cdot 30$ & 12.79 & $18 \cdot 47$ & $0 \cdot 00$ \\
\hline cy $17: 0$ & 0.00 & $0 \cdot 00$ & 0.00 & 0.00 & 0.00 & 0.00 & $0 \cdot 00$ & 0.00 & $5 \cdot 17$ & 0.00 & 0.00 & 0.00 & 0.00 & 0.0 & 0.00 & 0.00 & $0 \cdot 00$ \\
\hline $17: 0$ & 0.40 & 0.22 & $0 \cdot 22$ & 0.00 & 0.21 & 0.00 & 0.00 & 0.03 & 0.00 & 0.00 & 0.00 & $0 \cdot 25$ & $0 \cdot 00$ & $0 \cdot 3$ & $0 \cdot 25$ & $0 \cdot 00$ & 0.00 \\
\hline UNK 1 & 0.00 & $0 \cdot 00$ & 0.00 & $11 \cdot 87$ & 0.00 & 0.00 & 10.44 & 0.00 & 0.00 & 0.00 & 0.0 & 0.00 & $0 \cdot 00$ & 0.0 & $0 \cdot 00$ & $0 \cdot 00$ & 0.00 \\
\hline UNK 2 & 0.00 & $0 \cdot 00$ & $0 \cdot 00$ & $0 \cdot 00$ & 0.00 & 0.00 & $2 \cdot 63$ & 0.00 & 0.00 & 0.00 & 0.00 & 0.00 & $0 \cdot 00$ & 0.00 & $0 \cdot 00$ & $0 \cdot 00$ & 0.00 \\
\hline UNK 3 & 0.00 & 0.00 & $0 \cdot 00$ & 0.00 & $2 \cdot 23$ & 0.00 & $4 \cdot 62$ & 0.00 & 0.00 & 0.00 & 0.00 & 0.00 & $0 \cdot 00$ & $0 \cdot 0$ & $0 \cdot 00$ & 0.00 & 0.00 \\
\hline UNK 4 & 0.00 & 0.00 & $0 \cdot 00$ & $0 \cdot 23$ & 0.00 & 0.00 & $1 \cdot 12$ & 0.00 & 0.00 & 0.00 & 0.00 & 0.00 & $0 \cdot 00$ & 0.0 & $0 \cdot 00$ & $0 \cdot 00$ & 0.00 \\
\hline $18: 1 \omega 9 c$ & 0.00 & $0 \cdot 00$ & 0.00 & 0.00 & $3 \cdot 40$ & $32 \cdot 34$ & 0.00 & 0.00 & 0.00 & 0.00 & 0.00 & 0.00 & $0 \cdot 00$ & 0.00 & $0 \cdot 00$ & $0 \cdot 00$ & 0.00 \\
\hline$\omega 8 \mathrm{c}$ & 0.00 & 0.00 & 0.00 & 0.00 & 0.00 & $32 \cdot 28$ & $64 \cdot 68$ & $62 \cdot 02$ & 0.00 & 0.00 & 0.00 & $0 \cdot 00$ & 0.00 & 0.00 & $0 \cdot 00$ & 0.00 & 0.00 \\
\hline $18: 1 \omega 7 \mathrm{c}$ & 84.63 & $81 \cdot 06$ & $82 \cdot 76$ & $75 \cdot 12$ & $70 \cdot 41$ & $10 \cdot 48$ & $12 \cdot 53$ & $17 \cdot 35$ & 0.81 & 8.00 & $0 \cdot 0$ & $5 \cdot 03$ & 0.42 & $3 \cdot 4$ & 6.55 & 0.46 & $0 \cdot 25$ \\
\hline $18: 1 \omega 8 \mathrm{t}$ & 0.00 & 0.00 & 0.00 & $0 \cdot 00$ & 0.00 & $7 \cdot 57$ & 1.71 & $7 \cdot 50$ & 0.00 & 0.00 & 0.00 & 0.00 & $0 \cdot 00$ & 0.00 & $0 \cdot 00$ & $0 \cdot 00$ & 0.00 \\
\hline $18: 1 \omega 7 \mathrm{t}$ & 0.00 & 0.00 & $0 \cdot 68$ & 0.00 & 0.46 & 0.00 & $0 \cdot 00$ & 0.00 & $0 \cdot 14$ & 0.00 & 0.00 & 0.00 & 0.00 & 0.00 & $0 \cdot 00$ & 0.00 & 0.00 \\
\hline $18: 1 \omega 5 c$ & 0.00 & 0.00 & 0.00 & $0 \cdot 00$ & 0.36 & 0.00 & $0 \cdot 00$ & $0 \cdot 00$ & 0.00 & 0.00 & $0 \cdot 00$ & 0.00 & $0 \cdot 00$ & 0.00 & $0 \cdot 00$ & $0 \cdot 00$ & 0.00 \\
\hline $18: 0$ & $8 \cdot 61$ & 8.44 & $6 \cdot 88$ & $1 \cdot 01$ & $12 \cdot 20$ & 0.47 & $0 \cdot 36$ & $0 \cdot 66$ & $0 \cdot 21$ & 0.00 & 0.0 & $0 \cdot 40$ & $0 \cdot 27$ & 0.6 & 0.52 & $0 \cdot 29$ & $0 \cdot 00$ \\
\hline 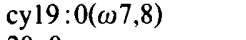 & 0.00 & 0.00 & 0.00 & 0.08 & $7 \cdot 35$ & 0.00 & 0.00 & $0 \cdot 00$ & $0 \cdot 00$ & 0.0 & $0 \cdot 0$ & 0.00 & $0 \cdot 00$ & $1 \cdot 5$ & 0.00 & $0 \cdot 00$ & 0.00 \\
\hline $20: 0$ & 0.00 & 0.00 & 0.00 & 0.00 & $0 \cdot 34$ & 0.00 & 0.00 & $0 \cdot 00$ & 0.00 & $0 \cdot 00$ & 0.00 & 0.00 & $0 \cdot 00$ & 0.00 & 0.00 & 0.00 & 0.00 \\
\hline$\Sigma \mu \mathrm{mol}(\mathrm{g} \text { dry } \mathrm{wt})^{-1}$ & 48.5 & $67 \cdot 24$ & $42 \cdot 51$ & 92.52 & $25 \cdot 03$ & $160 \cdot 02$ & $135 \cdot 04$ & $105 \cdot 35$ & $260 \cdot 96$ & $12 \cdot 08$ & $52 \cdot 74$ & 93.64 & $161 \cdot 07$ & $44 \cdot 88$ & 144.91 & $156 \cdot 81$ & 344.45 \\
\hline
\end{tabular}

Table 3. Characteristics of unknown FAME listed in Table 2 including equivalent chain lengths $(E C L)$ and $G C / M S$ diagnostic ions (positive electron impact)

Molecular ions $\left(\mathrm{M}^{+}\right)$were also verified by positive chemical ionization $\mathrm{GC} / \mathrm{MS}$.

\begin{tabular}{|c|c|c|c|c|c|}
\hline \multirow{2}{*}{$\begin{array}{l}\text { Unknown } \\
\text { FAME }\end{array}$} & \multicolumn{2}{|c|}{ ECL } & \multicolumn{3}{|c|}{ GC/MS Ions } \\
\hline & Rtx-1 & Rtx -225 & $\mathbf{M}^{+}$ & Base & Others \\
\hline UNK 1 & $17 \cdot 48$ & $18 \cdot 10$ & 294 & 55 & $262,245,220,209$ \\
\hline UNK 2 & $17 \cdot 53$ & $18 \cdot 15$ & 294 & 55 & $262,245,229,209$ \\
\hline UNK 3 & $17 \cdot 56$ & $18 \cdot 21$ & 294 & 67 & $262,245,220,209$ \\
\hline UNK 4 & $17 \cdot 60$ & $18 \cdot 19$ & - & 67 & $245,220,209$ \\
\hline
\end{tabular}

In group 1, the two literature strains of Methylobacterium organophilum XX (from Nichols et al., 1985) and the sample obtained for this study group very closely along with $M$. organophilum RG (from Nichols et al., 1985), M. extorquens AM1, and Methylobacterium sp. DM4 (SV $=0.90$, Fig. 1). All of these strains are pinkpigmented methylotrophs which use the serine pathway to assimilate formaldehyde (Table 1). This group was previously referred to as the 'type II' methylotrophs.

The other grouping of the serine methylotrophs was in group 2 and included the two replicates of Methylocystis parvus OBBP clustered at SV $=0.91$, and five cultures of Methylosinus trichosporium $\mathrm{OB} 3 \mathrm{~b}$ [including one literature value from Nichols et al. (1985)] grouped at $\mathrm{SV}=0 \cdot 82$.

The high- and low-oxygen incubation effects on membrane lipids of Methylococcus capsulatus BATH have been discussed by Jahnke \& Nichols (1986). The high-oxygen cultures formed one cluster $(\mathrm{SV}=0.93$ within group 4), whereas the low-oxygen culture was in group 3 with the strain grown by the University of Minnesota researchers $(\mathrm{SV}=0.82)$. The cultures produced for this study were grown for maximum biomass production, principally for $16 \mathrm{~S}$ rRNA sequence analysis. Although they were incubated with constant agitation (see Tsuji et al., 1990 for details), it is likely that this culture was also 'low-oxygen'. The other methylotrophic literature profile included in this analysis was that of Methylomonas sp. 761 (Nichols et al., 1985), which was most closely matched in group 5 to Methylomonas 


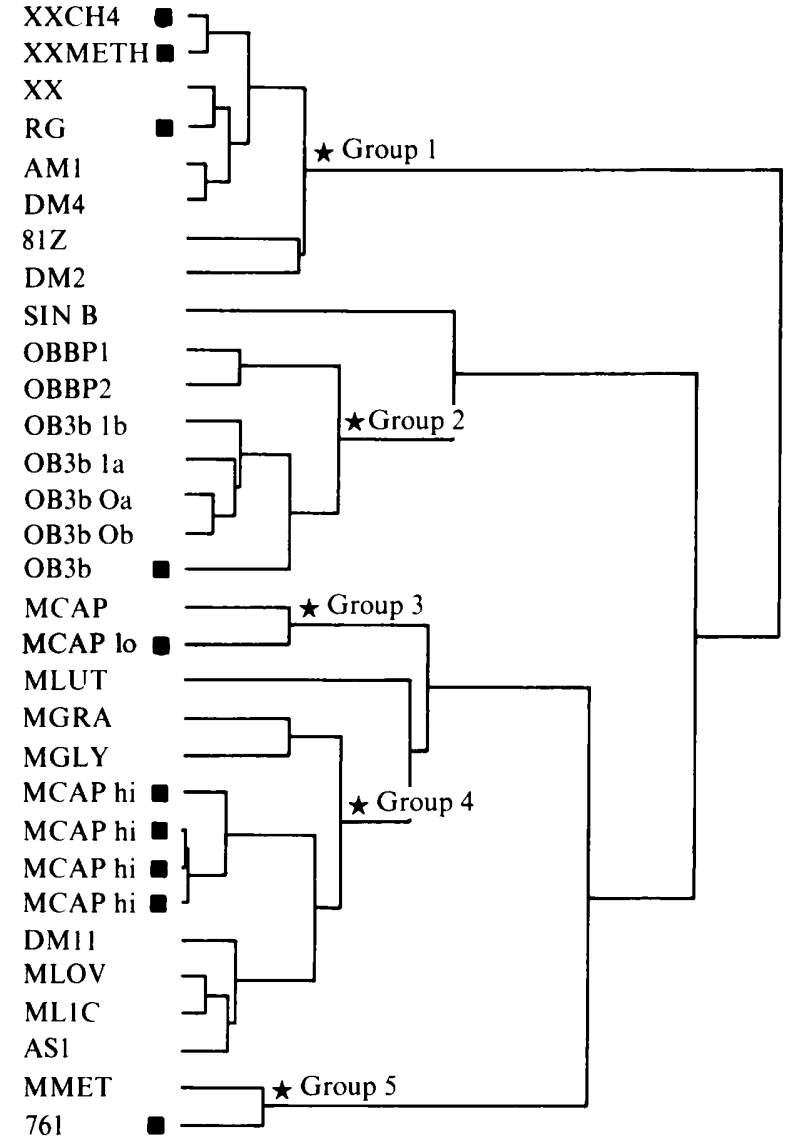

Fig. 1. Dendrogram of methylotrophic eubacterial PLFA profiles for strains detailed in the text. Values obtained from the literature are marked with a ' $\square$ ' symbol. Strain abbreviations are as shown in Table 1 and described in the text. Groups defined by an SV $>0.65$ are marked with a star and labelled for discussion purposes.

methanica $(\mathrm{SV}=0 \cdot 77)$, a pink-pigmented methylotroph which uses the ribulose monophosphate pathway to assimilate formaldehyde. This group was previously referred to as the 'type I' methylotrophs.

Based on the reproducibility of these phenotypic markers for replicates from different sources (Fig. 1), the PLFA profiles for the three XX strains, the two OBBP strains, the five OB3b strains, the two low-oxygen MCAP strains, and the four high-oxygen MCAP strains were separately averaged for further chemotaxonomic analyses.

\section{PLFA profiles and phenotype stability}

To date only rRNA sequences have been shown to provide a reliable bacterial phylogeny because of their universality, genetic stability, and conservation of structure (Murray et al., 1990). This phylogenetic analysis will not alone, however, provide conclusive taxonomic definitions for the eubacteria, and the importance of coupling these analyses to phenotypic descriptions is well established (Murray et al., 1990). Phenotypic characteristics will only be stable under environmental conditions which do not modify genotypic expression. PLFA profiles are no different. For instance, oxygen concentration has been shown to influence lipid profiles of Methylococcus capsulatus BATH (Jahnke \& Nichols, 1988; see Fig. 1). Not all changes in culture conditions will affect PLFA profiles, however, and PLFA changes that do occur will only occur within the range of biochemical modifications available to a particular genotype. Two examples are given below.

When Methylosinus trichosporium OB3b was grown in 0 and $1 \mu \mathrm{M}$-copper-supplemented medium, there were minimal PLFA profile changes (Table 4) although the addition of copper does promote significant physiological changes in this strain (R. S. Hanson, unpublished). Although not statistically significant, there was an increase in the trans/cis ratio on the addition of copper (Table 4). Since this is a well-documented indicator of physiological stress in eubacteria (see Guckert et al., 1986,1987 , and discussion below), further work is being conducted to attempt to correlate the observed physiological changes with membrane lipid stress markers (D. B. Ringelberg, D. C. White \& R. S. Hanson, unpublished).

Other eubacteria, like Agrobacterium tumefaciens, undergo what appear to be drastic PLFA changes during their normal growth phase. As shown in Table 4, when $A$. tumefaciens is in stationary growth phase, both the trans/cis and cyclopropyl/cis ratios for the $16: 1 \omega 7$ and $18: 1 \omega 7$ isomers significantly increase. Although these changes are significant, and would no doubt affect the classification of this species, they can also be predicted on the basis of known stress-induced biochemical modifications of the principal end products of eubacterial anaerobic desaturase fatty acid synthesis, $16: 1 \omega 7 \mathrm{c}$ and 18:1 $\omega 7$ c (see Guckert et al., 1986 and references within for review). These modifications include a methyl: donation across the $\omega 7$ c double bond from $S$-adenosylL-methionine catalysed by cyclopropyl synthetase to form the corresponding cyclopropyl ring, e.g. $16: 1 \omega 7 \mathrm{c} \rightarrow$ cy 17:0 $(\omega 7,8)$ (see Guckert et al., 1986 for discussion). Although this reaction is well characterized, it is not clear what benefit this metabolically-expensive stationary phase modification has to eubacterial survival. Even less is known about eubacterial trans PLFA synthesis, although the possibility of a constitutive isomerase along with preferential loss (degradation) of the cis isomer has been suggested as a mechanism for stress-induced increases in trans/cis ratios (Guckert et al., 1986, 1987). 
Table 4. PLFA profile changes with culture conditions

Results are expressed as mol\% of total recovered, with known eubacterial lipid stress ratios included. Details of culture conditions are given in the text.

\begin{tabular}{|c|c|c|c|c|}
\hline \multirow[b]{2}{*}{ Fatty acid } & \multicolumn{2}{|c|}{ Methylosinus trichosporium strain OB3b } & \multicolumn{2}{|c|}{ Agrobacterium tumefaciens (ATCC 33970) } \\
\hline & $\begin{array}{l}\text { Low copper growth } \\
(0 \mu \mathrm{M}-\mathrm{Cu})\end{array}$ & $\begin{array}{l}\text { High copper growth } \\
\qquad(1 \mu \mathrm{M}-\mathrm{Cu})\end{array}$ & $\begin{array}{l}\text { Exponential phase } \\
(<16 \mathrm{~h})\end{array}$ & $\begin{array}{l}\text { Stationary phase } \\
(>16 \mathrm{~h})\end{array}$ \\
\hline $14: 0$ & $0.02 \pm 0.03$ & $0.00 \pm 0.00$ & $0.15 \pm 0.13$ & $2.22 \pm 0.15 \dagger$ \\
\hline $\mathrm{i} 15: 0$ & $0.05 \pm 0.07$ & $0.00 \pm 0.00$ & $0.00 \pm 0.00$ & $0.00 \pm 0.00$ \\
\hline $15: 0$ & $0.00 \pm 0.00$ & $0.00 \pm 0.00$ & $0.00 \pm 0.00$ & $0.73 \pm 0.15 \dagger$ \\
\hline $16: 1 \omega 7 \mathrm{c}$ & $10.87 \pm 2.64$ & $10.56 \pm 0.75$ & $4.99 \pm 0.80$ & $0.48 \pm 0.19 \dagger$ \\
\hline $16: 1 \omega 7 \mathrm{t}$ & $0.10 \pm 0.07$ & $0.63 \pm 0.19$ & $0.02 \pm 0.03$ & $0.06 \pm 0.02$ \\
\hline $16: 0$ & $0.63 \pm 0.59$ & $1.72 \pm 0.40$ & $9.94 \pm 3.01$ & $19.31 \pm 2.03 \dagger$ \\
\hline i17:0 & $0.06 \pm 0.06$ & $0.05 \pm 0.07$ & $0.00 \pm 0.00$ & $0.00 \pm 0.00$ \\
\hline a17:0 & $0.10 \pm 0.08$ & $0 \cdot 10 \pm 0 \cdot 14$ & $0.00 \pm 0.00$ & $0.00 \pm 0.00$ \\
\hline $\operatorname{cy} 17: 0(\omega 7,8)$ & $0.00 \pm 0.00$ & $0.00 \pm 0.00$ & $2.00 \pm 1.74$ & $22 \cdot 15 \pm 0.76 \dagger$ \\
\hline $17: 0$ & $0.06 \pm 0.01$ & $0.00 \pm 0.00^{*}$ & $0.00 \pm 0.00$ & $0.06 \pm 0.04$ \\
\hline $18: 1 \omega 8 \mathrm{c}$ & $64.87 \pm 2.42$ & $59 \cdot 16 \pm 0 \cdot 16$ & $0.00 \pm 0.00$ & $0.00 \pm 0.00$ \\
\hline $18: 1 \omega 7 \mathrm{c}$ & $16.65 \pm 0.71$ & $18.06 \pm 4.78$ & $71.95 \pm 11.81$ & $4.68 \pm 1.76 \dagger$ \\
\hline $18: 1 \omega 8 t$ & $6.06 \pm 0.07$ & $8.93 \pm 5.54$ & $0.00 \pm 0.00$ & $0.00 \pm 0.00$ \\
\hline $18: 1 \omega 7 t$ & $0.00 \pm 0.00$ & $0.00 \pm 0.00$ & $0.00 \pm 0.00$ & $0.28 \pm 0.05 \dagger$ \\
\hline $18: 0$ & $0.53 \pm 0.16$ & $0.79 \pm 0.17$ & $0.60 \pm 0.08$ & $0.25 \pm 0.02 \dagger$ \\
\hline cy $19: 0(\omega 7,8)$ & $0.00 \pm 0.00$ & $0.00 \pm 0.00$ & $10 \cdot 35 \pm 6 \cdot 22$ & $49 \cdot 77 \pm 3 \cdot 22 \dagger$ \\
\hline \multicolumn{5}{|c|}{ Trans/cis ratios } \\
\hline $16: 1 \omega 7$ & $0.010 \pm 0.009$ & $0.061 \pm 0.022$ & $0.004 \pm 0.006$ & $0.128 \pm 0.016 \dagger$ \\
\hline $18: 1 \omega 7$ & $0 \pm 0$ & $0 \pm 0$ & $0 \pm 0$ & $0.065 \pm 0.016 \dagger$ \\
\hline $18: 1 \omega 8$ & $0.094 \pm 0.004$ & $0.151 \pm 0.093$ & $0 \pm 0$ & $0 \pm 0$ \\
\hline \multicolumn{5}{|c|}{ Cyclopropyl/cis ratios } \\
\hline $16: 1 \omega 7$ & $0 \pm 0$ & $0 \pm 0$ & $0 \cdot 37 \pm 0.32$ & $53 \cdot 00 \pm 24 \cdot 37^{*}$ \\
\hline $18: 1 \omega 7$ & $0 \pm 0$ & $0 \pm 0$ & $0 \cdot 16 \pm 0 \cdot 10$ & $12 \cdot 18 \pm 5 \cdot 57^{*}$ \\
\hline
\end{tabular}

* Change in PLFA content significant $(0.01<P<0.05)$ for changes in culture conditions.

$\dagger$ Change in PLFA content significant $(P<0 \cdot 01)$ for changes in culture conditions.

The commercially available Microbial Identification System (Microbial ID Inc., Newark, DE, USA), which is based on the analysis of whole-cell, ester-linked fatty acid profiles, recognizes the importance of phenotypic stability to library searching for identification of unknown strains. This procedure requires the unknown strains to be grown on defined media under specific conditions to provide the biomass for lipid analysis (Microbial Identification System Operating Manual, Version 3.0).

Since the Microbial Identification System is directed at the identification of clinical isolates, the incubation medium and conditions are generally not too restrictive. The reproducibility of the PLFA profiles described above (and in Fig. 1) suggests that these extreme precautions might not be required for methylotrophs; however, one does need to be cautious of culture conditions, especially the growth phase. It is suggested that exponential growth phase biomass be used whenever possible and that PLFA profiles are monitored for known modifications occurring during cell stress, such as cyclopropyl formation and high $(>0 \cdot 1)$ trans/cis ratios
(Guckert et al., 1986, 1987). Several methylotrophs have been shown to increase cyclopropyl/cis PLFA ratios in stationary phase cultures (Urakami \& Komagata, 1979).

\section{Integrating PLFA phenotype and phylogenetic relationships}

Taxonomic relationships for methylotrophic eubacteria have been reported utilizing 16S rRNA (Tsuji et al., 1990), 5S rRNA (Ando et al. 1989; Bulygina et al., 1990), DNA base composition with DNA-DNA homologies (Urakami et al., 1985; Hood et al., 1987), as well as multivariate analyses of characters based on morphology, physiology and biochemistry (Green \& Bousfield, 1982; Jenkins \& Jones, 1987). Most of these studies have not used the same strains discussed in this work; hence, integration of relationships is difficult. We have chosen, threfore, to interpret the PLFA phenotypic relationships by reference to the $16 \mathrm{~S}$ rRNA phylogenetic work of Tsuji et al. (1990), from whom the test strains used in the present study were obtained. 


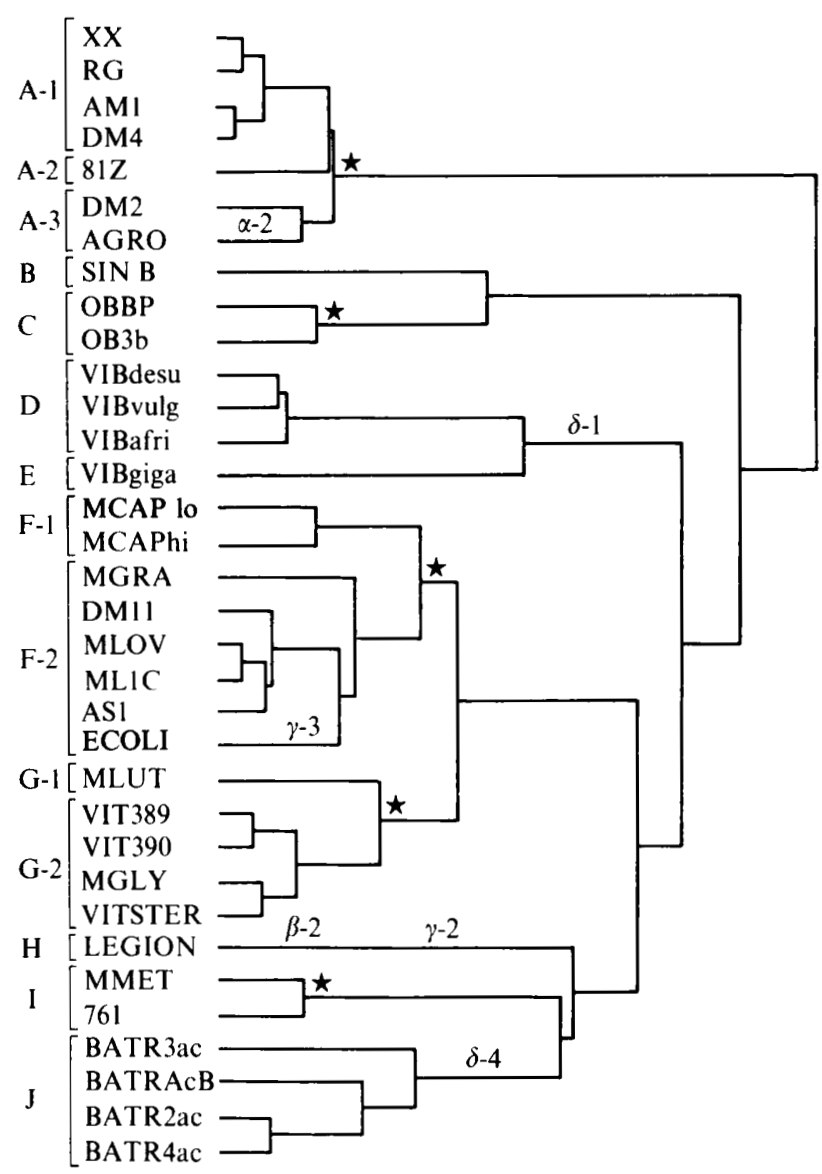

Fig. 2. Dendrogram of average methylotrophic PLFA profiles compared to non-methylotrophic species as described in the text. Groups (SV >0.65) are marked with a star. Clusters, as presented in Table 5, are also marked for discussion purposes. Strain abbreviations are as shown in Table 1 and described in the text. Other abbreviations are as follows: Desulfovibrio desulfuricans, VIBdesu; Desulfovibrio vulgaris, VIBvulg; Desulfovibrio africanus, VIBafri; Desulfovibrio gigas, VIBgiga; Escherichia coli, ECOLI; Vitreoscilla sp. 389, VIT389; Vitreoscilla sp. 390, VIT390; Vitreoscilla stercoraria, VITSTER; Legionella sp., LEGION; Desulfobacter sp. 3ac10, BATR3ac; Desulfobacter sp. AcBa, BATRAcB; Desulfobacter postgatei 2ac9, BATR2ac; Desulfobacter sp. 4ac11, BATR4ac.

In addition to having $16 \mathrm{~S}$ rRNA and PLFA results from the same methylotroph strains, we included PLFA profiles from several other eubacteria with wellestablished phylogenetic relationships. Fig. 2 shows the relationships for these Proteobacteria. Groups $(\mathrm{SV}>0.65)$ are marked with a star. Separate clusters are also marked and detailed in Table 5 with the strains, SV and a rank order listing of the mean $( \pm S D)$ for the characteristic PLFA from each cluster. In the left-hand column of Table 2, the 16S rRNA division is listed for the non-methylotrophic strains as reported in Woese (1987) or Devereux et al. (1989).

Tsuji et al. (1990) reported that the methylotrophs are not an evolutionarily coherent group, but are scattered throughout the $\alpha$-, $\beta$ - and $\gamma$-subdivisions of the Proteobacteria as defined by Woese (1987). The PLFA phenotypic analysis agrees with this conclusion. When non-methylotroph PLFA profiles from all subdivisions of the Proteobacteria $(\alpha-, \beta-, \gamma-, \delta$-) were included in the cluster analysis, methylotrophic strains grouped (SV > 0.65) with all eubacteria except members of the $\delta$-subdivision, the sulphate-reducing bacteria (SRB). PLFA profiles differentiated the SRB from other Proteobacteria as well as the Desulfovibrio spp. group $(\delta-1)$ from the Desulfobacter spp. group $(\delta-4)$. The representative PLFA profiles from the literature for these groups are listed in Table 5; however, these groups will not be discussed further since they are both phenotypically and phylogenetically distinct from the methylotrophs.

Phylogenetic analysis has indicated that all methylotrophs using the serine pathway ('type II') to assimilate formaldehyde fall into two groups within the $\alpha$-subdivision (Tsuji et al., 1990). One group included the pinkpigmented 'type II' methylotrophs and the other most of the non-pigmented 'type II' methylotrophs. PLFA analysis provided the same results. The 'type II' pigmented strains were tightly clustered (cluster A-1, Fig. 2) due to characteristically high proportions of $18: 1 \omega 7 \mathrm{c}$ (Table 5). Non-pigmented serine pathway methylotrophs were divided between clusters A-2 and A-3, which could be grouped (SV $>0.65$ ) with A-1, and clusters B and C (Fig. 2).

Agrobacterium tumefaciens is a common reference strain for phylogenetic discussions and has been established within the $\alpha-2$ subdivision (Woese, 1987). Its PLFA profile is included in the group of clusters A-1, A-2 and A-3 (Fig. 2), suggesting agreement between the PLFA and 16S rRNA taxonomic placement of the serine pathway methylotrophs in the $\alpha$ subdivision.

A potential discriminating feature of these pinkpigmented methylotrophs (cluster A-1) and the two most similar non-pigmented serine pathway methylotrophs $(81 \mathrm{Z}$ and DM2) is the level of cy $19: 0(\omega 7,8)$ (Table 5). As discussed above, this value may be influenced by culture conditions, so a definitive analysis of phenotypic relationships for this group would require strict attention to culture conditions. It is curious, though, that with over $80 \%$ of their PLFA as $18: 1 \omega 7 \mathrm{c}$, there was no cy 19:0 $(\omega 7,8)$ detected for any of the pink-pigmented methylotrophs using the serine pathway to assimilate formaldehyde (Table 2). Urakami \& Komagata (1979) also noted that not all methylotrophic species in their study increased their cyclopropyl content during stationary phase. The lack of cyclopropyl synthetase may, therefore, provide another key characteristic for differentiating this group.

Both the 16S rRNA and PLFA analyses divided the serine pathway methylotrophs into two groups. The 
Table 5. PLFA characteristics of clustered strains

\begin{tabular}{|c|c|c|c|c|c|}
\hline \multirow[t]{2}{*}{$\begin{array}{l}\text { 16S rRNA } \\
\text { division* }\end{array}$} & \multirow{2}{*}{$\begin{array}{l}\text { Strains grouped } \\
\text { Methylobacterium organophilum } \mathrm{XX} \\
\text { M. organophilum } \mathrm{RG} \\
\text { M. extorquens AM1 } \\
\text { Methylobacterium sp. DM4 }\end{array}$} & \multirow{2}{*}{$\begin{array}{l}\text { SV } \\
0.92\end{array}$} & \multicolumn{2}{|c|}{$\begin{array}{l}\text { Mol\% PLFA } \\
(\text { mean } \pm \text { SD })\end{array}$} & \multirow[t]{2}{*}{$\begin{array}{l}\text { Clusters from } \\
\text { Fig. } 1\end{array}$} \\
\hline & & & $\begin{array}{l}18: 1 \omega 7 \mathrm{c} \\
18: 0 \\
16: 0 \\
16: 1 \omega 7 \mathrm{c} \\
18: 1 \omega 7 \mathrm{t}\end{array}$ & $\begin{array}{r}(83.6 \pm 2.4) \\
(8.2 \pm 1.6) \\
(3.4 \pm 1.1) \\
(2.9 \pm 3.4) \\
(1.2 \pm 1.0)\end{array}$ & \\
\hline & Methylosinus methanica $81 \mathrm{Z}$ & & \multicolumn{2}{|c|}{ (see Table 2 for profile) } & A-2 \\
\hline \multirow[t]{3}{*}{$\alpha-2$} & $\begin{array}{l}\text { Hyphomicrobium sp. DM2 } \\
\text { Agrobacterium tumefaciens }\end{array}$ & $0 \cdot 86$ & $\begin{aligned} 18: 1 \omega 7 \mathrm{c} \\
\mathrm{cy} 19: 0(\omega 7,8) \\
16: 0 \\
18: 0 \\
16: 1 \omega 7 \mathrm{c}\end{aligned}$ & $\begin{array}{r}(71 \cdot 2 \pm 1 \cdot 1) \\
(8 \cdot 8 \pm 2 \cdot 1) \\
(6 \cdot 5 \pm 4 \cdot 9) \\
(6.4 \pm 8 \cdot 2) \\
(2 \cdot 5 \pm 3 \cdot 5)\end{array}$ & A-3 \\
\hline & Methylosinus sp. B & & \multicolumn{2}{|c|}{ (see Table 2 for profile) } & B \\
\hline & $\begin{array}{l}\text { Methylocystis parvus } \mathrm{OBBP} \\
\text { Methylosinus trichosporium } \mathrm{OB} 3 \mathrm{~b}\end{array}$ & $0 \cdot 83$ & $\begin{array}{l}18: 1 \omega 8 \mathrm{c} \\
18: 1 \omega 7 \mathrm{c} \\
16: 1 \omega 7 \mathrm{c} \\
\text { UNK } 1 \\
18: 1 \omega 7 \mathrm{t}\end{array}$ & $\begin{array}{r}(62 \cdot 1 \pm 3 \cdot 7) \\
(15 \cdot 7 \pm 4 \cdot 5) \\
(5 \cdot 5 \pm 6 \cdot 9) \\
(5 \cdot 2 \pm 7 \cdot 4) \\
(4 \cdot 8 \pm 4 \cdot 3)\end{array}$ & $\mathrm{C}$ \\
\hline $\begin{array}{l}\delta-1 \\
\delta-1 \\
\delta-1\end{array}$ & $\begin{array}{l}\text { Desulfovibrio desulfuricans } \\
\text { D. vulgaris } \\
\text { D. africanus }\end{array}$ & $0 \cdot 88$ & $\begin{array}{l}\text { i1 } 17: 1 \omega 7 \mathrm{c} \\
16: 0 \\
\text { i15:0 } \\
16: 1 \omega 7 \mathrm{c} \\
\text { i1 } 17: 0 \\
\text { a15:0 } \\
18: 0 \\
18: 1 \omega 7 \mathrm{c} \\
\text { a17:0 } \\
\text { a17:1 } 1 \omega 7 \mathrm{c}\end{array}$ & $\begin{array}{r}(28.7 \pm 3.9) \\
(18.0 \pm 3.8) \\
(16.3 \pm 4.1) \\
(8.5 \pm 2.5) \\
(8.2 \pm 0.2) \\
(3.5 \pm 1.6) \\
(3.1 \pm 2.2) \\
(2.9 \pm 2.2) \\
(2.5 \pm 1.2) \\
(2.3 \pm 2.0)\end{array}$ & $\mathrm{D}$ \\
\hline \multirow[t]{3}{*}{$\delta-1$} & Desulfovibrio gigas & & \multicolumn{2}{|c|}{ (see Edlund et al., 1985 for profile) } & $\mathbf{E}$ \\
\hline & $\begin{array}{c}\text { Methylococcus capsulatus BATH } \\
\text { grown under low and high } \mathrm{O}_{2}\end{array}$ & 0.84 & $\begin{array}{c}16: 0 \\
16: 1 \omega 7 \mathrm{c} \\
16: 1 \omega 6 \mathrm{c} \\
16: 1 \omega 5 \mathrm{c} \\
14: 0 \\
\mathrm{cy} 17: 0(\omega 7,8) \\
\mathrm{cy} 17: 0(\omega 6,7)\end{array}$ & $\begin{array}{r}(39.5 \pm 3.6) \\
(27.1 \pm 6.7) \\
(10.5 \pm 3.4) \\
(7.9 \pm 2 \cdot 7) \\
(4.5 \pm 2 \cdot 2) \\
(3.8 \pm 3 \cdot 9) \\
(3.4 \pm 5 \cdot 3)\end{array}$ & $F-1$ \\
\hline & $\begin{array}{l}\text { Methylomonas gracilis } \\
\text { Methylotrophic sp. DM11 } \\
\text { Methylomonas methylovora } \\
\text { Methylomonas methanolica } \\
\text { Methylophilus methylotrophus AS1 } \\
\text { E. coli }\end{array}$ & $0 \cdot 77$ & $\begin{array}{c}16: 0 \\
16: 1 \omega 7 \mathrm{c} \\
\text { cy } 17: 0(\omega 7,8) \\
18: 1 \omega 7 \mathrm{c}\end{array}$ & $\begin{array}{r}(43 \cdot 3 \pm 4 \cdot 7) \\
(38.5 \pm 5 \cdot 0) \\
(10 \cdot 4 \pm 6 \cdot 7) \\
(4 \cdot 5 \pm 6 \cdot 2)\end{array}$ & $\mathrm{F}-2$ \\
\hline$\gamma-3$ & Methylococcus luteus & & \multicolumn{2}{|c|}{ (see Table 2 for profile) } & $\mathrm{G}-1$ \\
\hline$\beta-2$ & $\begin{array}{l}\text { Methylobacillus glycogenes } \\
\text { Vitreoscilla stercoraria } \\
\text { Vitreoscilla spp. strains } 389,390\end{array}$ & 0.87 & $\begin{array}{l}16: 1 \omega 7 \mathrm{c} \\
16: 0 \\
18: 1 \omega 7 \mathrm{c} \\
14: 0\end{array}$ & $\begin{array}{r}(52.9 \pm 2.9) \\
(30.8 \pm 4 \cdot 1) \\
(7.5 \pm 1 \cdot 8) \\
(5 \cdot 3 \pm 4 \cdot 3)\end{array}$ & G-2 \\
\hline$\gamma-2$ & Legionella sp. & & $\begin{array}{l}\text { i1 } 16: 0 \\
\text { a15:0 } \\
\text { a17:0 } \\
16: 1 \omega 7 \mathrm{c} \\
\text { i16:1 } \\
\text { a17:1 } \\
16: 0 \\
18: 0\end{array}$ & $\begin{array}{l}(34.6 \pm 3.0) \dagger \\
(19.1 \pm 3.3) \\
(15.0 \pm 2.4) \\
(11.5 \pm 1.0) \\
(7.3 \pm 0.4) \\
(2.8 \pm 0.5) \\
(1.4 \pm 0.4) \\
(1.0 \pm 0.2)\end{array}$ & $\mathbf{H}$ \\
\hline
\end{tabular}


Table 5-continued

\begin{tabular}{|c|c|c|c|c|c|}
\hline \multirow[t]{2}{*}{$\begin{array}{l}\text { 16S rRNA } \\
\text { division* }\end{array}$} & \multirow{2}{*}{$\begin{array}{l}\text { Strains grouped } \\
\text { Methylomonas methanica } \\
\text { Methylomonas sp. } 761\end{array}$} & \multirow{2}{*}{$\frac{\text { SV }}{0 \cdot 86}$} & \multicolumn{2}{|c|}{$\begin{array}{c}\text { Mol\% PLFA } \\
(\text { mean } \pm \text { SD) }\end{array}$} & \multirow[t]{2}{*}{$\begin{array}{c}\text { Clusters from } \\
\text { Fig. } 1\end{array}$} \\
\hline & & & $\begin{array}{l}16: 1 \omega 8 \mathrm{c} \\
14: 0 \\
16: 1 \omega 7 \mathrm{c} \\
16: 1 \omega 5 \mathrm{c} \\
16: 1 \omega 5 \mathrm{t} \\
16: 0 \\
16: 1 \omega 7 \mathrm{t} \\
16: 1 \omega 6 \mathrm{c}\end{array}$ & $\begin{array}{r}(35.4 \pm 7.7) \\
(19.3 \pm 2.0) \\
(14.3 \pm 4.9) \\
(8.6 \pm 10.9) \\
(7.9 \pm 10.9) \\
(5.6 \pm 1.9) \\
(3.8 \pm 2.6) \\
(1.7 \pm 2.4)\end{array}$ & \\
\hline $\begin{array}{l}\delta-4 \\
\delta-4 \\
\delta-4 \\
\delta-4\end{array}$ & $\begin{array}{l}\text { Desulfobacter } \mathrm{sp} .3 \mathrm{ac} 10 \\
\text { Desulfobacter } \mathrm{sp} . \text { AcBa } \\
\text { Desulfobacter postgatei } 2 \mathrm{ac} 9 \\
\text { Desulfobacter } \mathrm{sp} .4 \mathrm{ac} 11\end{array}$ & 0.67 & 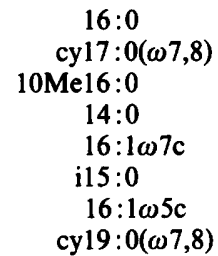 & $\begin{array}{r}(27.0 \pm 6.3) \\
(22.2 \pm 10.4) \\
(15.4 \pm 6.9) \\
(15.2 \pm 10.8) \\
(4.8 \pm 2.6) \\
(3.4 \pm 3.6) \\
(1.8 \pm 0.2) \\
(1.3 \pm 1.5)\end{array}$ & $\mathbf{J}$ \\
\hline
\end{tabular}

* After Woese (1987) and Devereux et al. (1989).

$\dagger$ Variability for group as reported by Pfiffner et al. (1988).

second PLFA group was characterized by PLFA which include $18: 1 \omega 8 \mathrm{c}$. These strains were Methylosinus sp. B (in cluster B), Methylocystis parvus OBBP and Methylosinus trichosporium $\mathrm{OB} 3 \mathrm{~b}$ (both in cluster $\mathrm{C}$ ). The membrane lipids of $\mathrm{OB} 3 \mathrm{~b}$ have been the subject of several studies, in fact the results of Nichols et al. (1985) are included in this analysis (Fig. 1). We have emphasized the importance of adequate analytical verification of PLFA structure for this type of analysis by pointing out that in an initial description of the membrane fatty acids of OB3b (Weaver et al., 1975) the 18:1 isomers were not separated. Note that if all $18: 1$ isomers were not resolved for this analysis, the cluster of OBBP and $\mathrm{OB} 3 \mathrm{~b}$ (cluster $\mathrm{C}$ ) would contain approximately $78 \% 18: 1$ and would not be separable from the pink-pigmented 'type II' methylotrophs (Table 5).

The 16S rRNA phylogenetic analysis for the type I' (ribulose monophosphate pathway) methylotrophs suggested a split into the $\beta$ - and $\gamma$-subdivisions of the Proteobacteria (Tsuji et al., 1990). 'Type I' methylotrophs are generally characterized by a dominance of 16:1 isomers with few PLFA of any longer chain lengths (Nichols et al., 1985). The PLFA analysis indicated two principal groups either clustered with $E$. coli $(\gamma-3$, cluster F-2) or Vitreoscilla stercoraria ( $\beta-2$, cluster G-2) (Fig. 2) with some outliers.

One of these outliers was Methylococcus capsulatus BATH (MCAP, cluster F-1). The physiology of this organism is apparently not completely resolved and it has been described as a 'type X' methylotroph (Whittenbury \& Dalton, 1980; Tsuji et al., 1990). The PLFA phenotype of this strain is unusual due to the presence of the $16: 1 \omega 6 \mathrm{c}$ isomer (Table 5), regardless of the oxygen content of the culture medium (Jahnke \& Nichols, 1986).

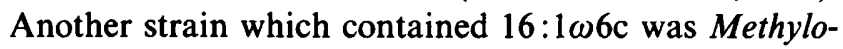
monas methanica (MMET, Table 2), a pink-pigmented ribulose monophosphate methylotroph (Table 1). The 16S rRNA phylogenetic analysis suggested that both of these strains fall into the $\gamma$ subdivision, although sequence differences between the two were over $20 \%$ (Tsuji et al., 1990). Although they both have the unusual $16: 1 \omega 6 \mathrm{c}$, there is a clear separation by PLFA analysis. MCAP is most similar to the group of methylotrophs grouped with $E$. coli, a $\gamma-3$ organism. MMET is most closely related to another Methylomonas strain, 761 (see Nichols et al., 1985) due to its diversity of $16: 1$ isomers: $16: 1 \omega 8 \mathrm{c}, \quad 16: 1 \omega 7 \mathrm{c}, \quad 16: 1 \omega 7 \mathrm{t}, \quad 16: 1 \omega 6 \mathrm{c}, \quad 16: 1 \omega 6 \mathrm{t}$, $16: 1 \omega 5 \mathrm{c}$ and $16: 1 \omega 5 \mathrm{t}$ (Table 5).

The other ribulose monophosphate obligate methylotrophs included in the 16S rRNA tree of Tsuji et al. (1990) were Methylophilus methylotrophus AS1 and methylotrophic DM11. These were found to be most closely phylogenetically related to eubacteria in the $\beta-3$ subdivision. The PLFA phenotypes for these strains are also very similar (Table 5) and group with the other ribulose monophosphate methylotrophs (cluster I, Fig. 2); Methylomonas gracilis, Methylomonas methylovora and Methylomonas methanolica. This group of methylotrophs, however, had a profile similar to that of the $\gamma-3$ $E$. coli, rather than a $\beta$-subdivision eubacterium like $V i t r e o s c i l l a$. Woese (1987) notes that the $\beta$ - and $\gamma$-subdivisions are very closely related and may, in fact, be branches of the same, larger subdivision. Additional PLFA profiles from these subdivisions of the Proteo- 
bacteria are needed to help determine these relationships.

In conclusion, we have reported PLFA profiles for 17 methylotrophs with sufficient analytical detail for use in future taxonomic evaluations. The ability to include such literature data for multivariate analyses was demonstrated. Cluster analysis of PLFA profiles generated by separate investigators with different culture conditions indicated reproducibility by strain and species. The methylotroph data have shown that these profiles are not 'inadequate for varieties of micro-organisms with similar metabolic traits' (Tsuji et al., 1990). The phenotypic relationships described here compare favourably with phylogenetic associations based on 16S rRNA data for these same strains (Tsuji et al., 1990), and will continue to be a valuable phenotypic marker for Proteobacteria taxonomy.

Mr Jonathan Johnston is acknowledged for his PLFA profile analysis of these strains as well as assistance with $\mathrm{GC} / \mathrm{MS}$ verification of FAME structure. Ms Jennifer Loukota and Mr Kevin Kroeger conducted the Agrobacterium growth phase experiment. Portions of this project were supported by the University of Tennessee Science Alliance, the Gas Research Institute (GRI 5086-260-1303), the Office of Naval Research (ONR N00014-88-K-0489) and the Subsurface Science Program, Office of Health and Environmental Research, Department of Energy (DOE DE-FG05-90ER60988).

\section{References}

Ando, S., Kato, S.-I. \& Komagata, K. (1989). Phylogenetic diversity of methanol-utilizing bacteria deduced from their $5 \mathrm{~S}$ ribosomal RNA sequences. Journal of General and Applied Microbiology 35, 351-361.

BLIGH, E. G. \& DYER, W. M. (1959). A rapid method of lipid extraction and purification. Canadian Journal of Biochemistry and Physiology 35, 911-917.

Brondz, I. \& OlSEN, I. (1986). Microbial chemotaxonomy. Chromatography, electrophoresis, and relevant profiling techniques. Journal of Chromatography 379, 367-411.

Bulygina, E. S., Galchenko, V. F., Govorukhina, N. I., Netrusov, A. I., Nikitin, D. I., Trotsenko, Y. A. \& Chumakov, K. M. (1990). Taxonomic studies on methylotrophic bacteria by $5 \mathrm{~S}$ ribosomal RNA sequencing. Journal of General Microbiology 136, 441-446.

Christie, W. W. (1989). Gas Chromatography and Lipids. Ayr : The Oily Press.

Dalton, H. \& WhitTenbury, R. (1976). The acetylene reduction technique as an assay for nitrogenase activity in the methane oxidizing bacterium Methylococcus capsulatus BATH. Archives of Microbiology 109, 147-151.

Devereux, R., Delaney, M., Widdel, F. \& Stahl, D. A. (1989). Natural relationships among sulfate-reducing eubacteria. Journal of Bacteriology 171, 6689-6695.

Dowling, N. J. E., WIDdel, F. \& White, D. C. (1986). Phospholipid, ester-linked fatty acid biomarkers of acetate-oxidizing sulphatereducers and other sulfide-forming bacteria. Journal of General Microbiology 132, 1815-1825.

Edlund, A., Nichols, P. D., Roffey, R. \& White, D. C. (1985). Extractable and lipopolysaccharide fatty acid and hydroxy acid profiles from Desulfovibrio species. Journal of Lipid Research 26, 982-988.

GoOdFellow, M. \& Minnikin, D. E. (editors) (1985). Chemical Methods in Bacterial Systematics. London: Academic Press.
Green, P. N. \& Bousfield, I. J. (1982). A taxonomic study of some Gram-negative facultatively methylotrophic bacteria. Journal of General Microbiology 128, 623-638.

GUCKERT, J. B. \& WHITE, D. C. (1988). Phospholipid, ester-linked fatty acid analysis in microbial ecology. Proceedings of the Fourth International Symposium of Microbial Ecology, pp. 455-459. Edited by F. Megusar \& M. Gantor. Ljubljana: Slovene Society for Microbiology.

Guckert, J. B., ANTWorth, C. P., Nichols, P. D. \& White, D. C. (1985). Phospholipid, ester-linked fatty acid profiles as reproducible assays for changes in prokaryotic community structure of estuarine sediments. FEMS Microbiology Ecology 31, 147-158.

GuCKert, J. B., Hood, M. A. \& WhITE, D. C. (1986). Phospholipid ester-linked fatty acid profile changes during nutrient deprivation of Vibrio cholerae: increases in the trans/cis ratio and proportions of cyclopropyl fatty acids. Applied and Environmental Microbiology 52, 794-801.

Guckert, J. B., Ringelberg, D. B. \& White, D. C. (1987). Biosynthesis of trans fatty acids from acetate in the bacterium, Pseudomonas atlantica. Canadian Journal of Microbiology 33, 748-754.

Hood, D. W., Dow, C. S. \& Green, P. N. (1987). DNA:DNA hybridization studies on the pink-pigmented facultative methylotrophs. Journal of General Microbiology 133, 709-720.

JAHNKE, L. L. \& Nichols, P. D. (1986). Methyl sterol and cyclopropane fatty acid composition of Methylococcus capsulatus grown at low oxygen tensions. Journal of Bacteriology 167, 238-242.

JeNKINS, O. \& JoNES, D. (1987). Taxonomic studies of some Gramnegative methylotrophic bacteria. Journal of General Microbiology 133, 453-473.

KATES, M. (1986). Techniques of Lipidology: Isolation, Analysis and Identification of Lipids, 2nd revised edn. Amsterdam: Elsevier.

Murray, R. G. E., Brenner, D. J., Colwell, R. R., De Vos, P., Goodfellow, M., Grimont, P. A. D., Pfennig, N., StackeBRANDT, E. \& Zavarzin, G. A. (1990). Report of the ad hoc committee on approaches to taxonomy within the Proteobacteria. International Journal of Systematic Bacteriology 40, 213-215.

Nichols, P. D., Smith, G. A., ANTworth, C. P., Hanson, R. S. \& WHITE, D. C. (1985). Phospholipid and lipopolysaccharide normal and hydroxy fatty acids as potential signatures for methaneoxidizing bacteria. FEMS Microbiology Ecology 31, 327-335.

Nichols, P. D., GuCKeRT, J. B. \& WhITE, D. C. $(1986 a)$. Determination of monosaturated fatty acid double-bond position and geometry for microbial monocultures and complex consortia by capillary GC-MS of their dimethyl disulphide adducts. Journal of Microbiological Methods 5, 49-55.

Nichols, P. D., StulP, B. K., Jones, J. G. \& White, D. C. (1986b). Comparison of fatty acid content and DNA homology of the filamentous gliding bacteria Vitreoscilla, Flexibacter, Filibacter. Archives of Microbiology 146, 1-6.

Pfiffner, S. M., Ringelberg, D. B., Tunlid, A. \& Tyndall, R. L. (1988). Detection of Legionella infection of Naegleria by phospholipid fatty acid biomarkers. Abstracts of the Annual Meeting of the American Society for Microbiology Q-29, Miami Beach, Fl, 8-13 May, 1988.

Ratledge, C. \& Wilkinson, S. G. (editors) (1988). Microbial Lipids. London: Academic Press.

Ringelberg, D. B., Davis, J. D., Smith, G. A., Pfiffner, S. M., Nichols, P. D., Nickels, J. S., Henson, J. M., Wilson, J. T., Yates, M., Kampbell, D. H., Read, H. W., Stocksdale, T. T. \& White, D. C. (1989). Validation of signature polar lipid fatty acid biomarkers for alkane-utilizing bacteria in soils and subsurface aquifer materials. FEMS Microbiology Ecology 62, 39-50.

TORNABENE, T. G. (1985). Lipid analysis and the relationship to chemotaxonomy. Methods in Microbiology 18, 209-234.

Tsien, H. C., Bratina, B. J., Tsuji, K. \& Hanson, R. S. (1990). Use of oligodeoxynucleotide signature probes for identification of physiological groups of methylotrophic bacteria. Applied and Environmental Microbiology 56, 2858-2865.

Tsuit, K., Tsien, H. C., Hanson, R. S., DePalma, S. R., Scholtz, R. \& LAROCHE, S. (1990). 16S ribosomal RNA sequence analysis for 
determination of phylogenetic relationship among methylotrophs. Journal of General Microbiology 136, 1-10.

Urakami, T. \& Komagata, K. (1979). Cellular fatty acid composition and coenzyme $\mathrm{Q}$ system in Gram-negative methanol-utilizing bacteria. Journal of General and Applied Microbiology 25, 343-360.

Urakami, T., Tamaoka, J. \& Komagata, K. (1985). DNA base composition and DNA-DNA homologies of methanol-utilizing bactria. Journal of General and Applied Microbiology 31, 243-253.

Weaver, T. L., Patrick, M. A. \& Dugan, P. R. (1975). Whole-cell and membrane lipids of the methylotrophic bacterium Methylosinus trichosporium. Journal of Bacteriology 124, 602-605

White, D. C., Bobbie, R. J., Herron, J. S., King, J. D. \& Morrison, S. J. $(1979 a)$. Biochemical measurements of microbial mass and activity from environmental samples. In Native Aquatic Bacteria.
Enumeration, Activity and Ecology, ASTM STP 695, pp. 69-81. Edited by J. W. Costerton \& R. R. Colwell. Philadelphia: American Society for Testing and Materials.

White, D. C., Davis, W. M., Nickels, J. S., King, J. D. \& Bobbie, R. J. (1979b). Determination of sedimentary microbial biomass by extractable lipid phosphate. Oecologia 40, 51-62.

WoEse, C. R. (1987). Bacterial evolution. Microbiological Reviews 51, 221-271.

Wold, S., Albano, C. A., Dunn, W. J., III, Edlund, U., Esbensen, K., Geladi, P., Hellberg, S., Johansson, E., Lindberg, W. \& SЈöströM, M. (1984). Multivariate data analysis in chemistry. In Proceedings NATO Advanced Study Institute on Chemometrics, pp. 1-79. Edited by B. R. Kowalski. Dordrecht, Holland: Reidel Publishing Co. 